\title{
Preliminary Surgical Results of Single-Incision Transumbilical Laparoscopic Bariatric Surgery
}

\author{
Chih-Kun Huang • Jui-Chi Tsai • Chi-Hsien Lo • \\ Jer-Yiing Houng • Yaw-Sen Chen • Shu-Ching Chi • \\ Po-Huang Lee
}

Received: 01 October 2009 / Accepted: 30 December 2009/Published online: 30 January 2010

(C) The Author(s) 2010. This article is published with open access at Springerlink.com

\begin{abstract}
Background Recently, single-incision laparoscopic surgery (SILS) has been used for bariatric procedures, and this surgery is considered a type of minimally invasive surgery. When SILS is performed via the transumbilical route, the resultant abdominal wound is hidden and the cosmetic outcome is better. However, because of the small angle of manipulation and difficulty in liver traction, this technique is not used to perform complex bariatric surgery. In this prospective study, we used our novel technique, which involves the use of a liver-suspension tape and umbilicoplasty of an omega-shaped incision (omega umbilicoplasty), to perform laparoscopic bariatric surgery via the single-incision transumbilical (SITU) approach. We then assessed the safety and effectiveness of our surgical technique.

Methods We started performing and developing this technique from December 2008. Until July 2009, 40 consecutive patients underwent 40 bariatric procedures: two
\end{abstract}

C.-K. Huang $(\bowtie) \cdot$ Y.-S. Chen $\cdot$ P.-H. Lee

Department of General Surgery, E-Da Hospital,

1 E-Da Road, Jiau-shu Tseun, Yan-chau Shiang,

Kaohsiung County 824, Taiwan

e-mail: dr.ckhuang@hotmail.com

C.-K. Huang · J.-Y. Houng

Department of Chemical Engineering and Institute

of Biotechnology and Chemical Engineering, I-Shou University,

Kaohsiung County, Taiwan

C.-K. Huang $\cdot$ J.-C. Tsai $\cdot$ C.-H. Lo

Bariatric \& Metabolic International Surgery Center,

E-Da Hospital,

Kaohsiung, Taiwan

S.-C. Chi

Department of Nursing, E-Da Hospital,

Kaohsiung, Taiwan adjustable gastric band placements, six sleeve gastrectomies, and 32 Roux-en-Y gastric bypass operations, including five cases where concomitant cholecystectomy was performed. Results The mean operation time was $93.4 \mathrm{~min}$ and the mean duration of postoperative hospitalization was 1.15 days. No perioperative or postoperative complications or deaths occurred. Most patients were very satisfied with the cosmetic outcomes.

Conclusion Our technique can be safely and effectively used for SITU laparoscopic bariatric surgery. This technique will soon be used for advanced abdominal surgeries besides bariatric ones.

Keywords Minimally invasive surgery · Bariatric surgery · Gastric bypass - Cholecystectomy -

Single-incision transumbilical laparoscopic surgery .

Single-incision laparoscopic surgery

\section{Introduction}

Laparoscopic surgery was introduced about 100 years ago, and the concept of minimally invasive surgery (MIS) has dramatically altered the field of surgery. In classic laparoscopic surgery, five to seven incisions are required for the placement of multiple trocars. Surgeons have attempted to reduce the number and size of incisions or to develop new operations that can be performed using natural orifice translumenal endoscopic surgery (NOTES), which is considered to be a landmark technique in MIS [1]. Since the introduction of NOTES in 2004, numerous reports have described surgical interventions that can be performed using this technique [2, 3]. While this technique has garnered the interest of surgeons worldwide, the procedures and instruments used in it are still in the preliminary stages 
of investigation. Recently, single-incision laparoscopic surgery (SILS) has been used in several operations, including various urological and upper gastrointestinal and lower gastrointestinal operations [4-13]. The cosmetic outcomes of SILS are expected to be better when the operation is performed through the umbilicus. This is because the surgical wound is hidden within the umbilicus, leaving no visible abdominal scars. Recently, a new product, the Covidien SILS ${ }^{\text {TM }}$ Port (Connecticut, USA), has been developed for laparoscopic surgery. However, when this port is introduced through a $2.0-\mathrm{cm}$ umbilical incision, $<1.0 \mathrm{~cm}$ of free space is left between the cannulas and the manipulation of laparoscopic instruments is difficult because of crowding. Hence, the use of this port has been confined to simple procedures such as appendectomy and cholecystectomy. Furthermore, liver retraction, which is required during most bariatric surgeries to avoid the blocking of the surgical field by the liver, cannot be achieved with single-incision transumbilical (SITU) laparoscopic surgery. To extend the applicability of SILS to more advanced procedures, we developed a novel SITU laparoscopic surgery involving umbilicoplasty of an omega-shaped incision (hereafter, referred to as omega umbilicoplasty) and a liver-suspension tape (LST) and used this technique to perform different types of bariatric surgeries.

\section{Materials and Methods}

From December 2008, we started performing and developing SITU laparoscopic surgery in E-Da Hospital. The patients were fully informed about the procedures, and informed consent was obtained from them. A total of 40 morbidly obese patients were enrolled in this study, and until July 2009, a total of 40 SITU laparoscopic procedures were performed on these patients, including adjustable gastric band placement, sleeve gastrectomy, Roux-en-Y gastric bypass (RYGB), and RYGB with concomitant cholecystectomy. The surgical indications for bariatric surgery adhered to the 1991 National Institutes of Health consensus [14]. Concomitant cholecystectomy was performed if ultrasonographic evidence of gallstones was present. All procedures were performed using similar surgical techniques that involved omega umbilicoplasty and the use of LST. The time required for placing the LST and the total operation time, trocar to trocar, were recorded for all patients. Postoperative morphine use, length of hospitalization, and occurrence of complications were also recorded and analyzed (Table 1).

\section{Operative Technique}

The patient was placed in the supine position with the arms extended laterally. An anesthesiologist induced general
Table 1 Demographic characteristics of the study patients

Patient characteristic $(n=40)$

Mean age, years (range)

$29(19-53)$

Female/male ratio

$32: 8$

BMI $\left(\mathrm{kg} / \mathrm{m}^{2}\right)$

$40.1(35-52.3)$

Comorbid conditions, $n(\%)$

Hyperlipidemia

$17(42.5)$

NASH

$11(27.5)$

Hyperuricemia

$8(20.0)$

Hypertension

$7(17.5)$

Diabetes mellitus

$6(15.0)$

Gallstones

5 (12.5)

Osteoarthritis

$1(2.5)$

anesthesia and performed endotracheal intubation. For most operations, the surgeon stood on the right side of the patient and the assistant on the left. A 6-cm-long omega-shaped incision was made around the upper half of the umbilicus (Fig. 1a). The incision was deepened to the linea alba after dissecting the subcutaneous fat, and a 12- or 15-mm Versaport plus V2 trocar (Covidien) was inserted into the arch of the omega-shaped incision after establishing pneumoperitoneum, produced by carbon dioxide insufflation at a pressure of $15 \mathrm{mmHg}$. A $10-\mathrm{mm}$-long, rigid, $30^{\circ}$ video laparoscope was then inserted. Under direct visualization, two Versaport plus V2 trocars (sizes, 5 and $11 \mathrm{~mm}$ ) with fixation cannulas (Covidien) were inserted through both arms of the omega incision (Fig. 1b). We then inserted the LST into the peritoneal cavity. To prepare the LST, the length of the liver lobe was measured intraoperatively and a Jackson-Pratt drain tube was cut to the same length near the site of the drainage hole. Subsequently, 2-0 prolene sutures (monofilament polypropylene suture W8400; Ethicon) were inserted along the holes of the drain tube and the two needles attached to the sutures were retained for liver puncture (Fig. 2). One needle was placed in a needle holder, inserted into the lateral edge of the left liver lobe, and brought out through the abdominal wall in the left upper quadrant; the other needle was inserted into the left liver lobe near the falciform ligament and then brought out through the abdominal wall in the right upper quadrant. The liver was then retracted to an appropriate position, and the sutures were suspended with clamps (Fig. 2). If one LST was not sufficient to suitably retract the liver, we used another LST. During the cholecystectomies, the right liver lobe was similarly retracted with the LST if the Calot triangle was difficult to visualize and if dissection without countertraction was tedious. The tape was inserted into the edge of the right liver lobe, and the liver was retracted upward and in the cephalic direction. After achieving the appropriate liver traction, we commenced the surgical procedure. For SITU-laparoscopic Roux-en-Y gastric bypass 
Fig. 1 a A horizontal 6-cm-long omega-shaped skin incision around the upper half of the umbilicus; $\mathbf{b}$ triangular positioning of and distance between trocars
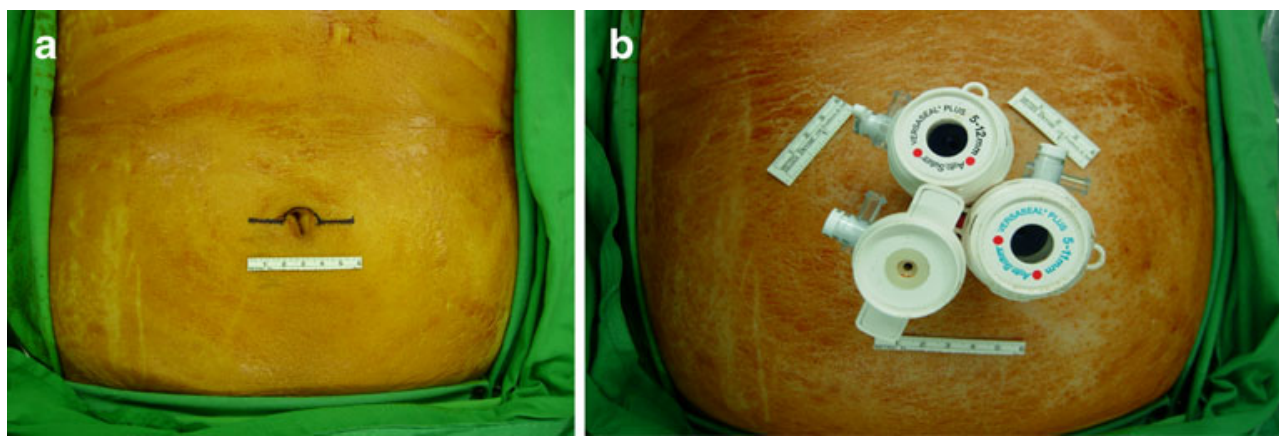

(LRYGB), we used laparoscopic linear staplers (Endo GIATM Universal Staplers XL, Covidien) to divide the stomach and form a 25-cc gastric pouch and 2.5-cm-long gastrojejunostomy. Next, jejunojejunostomy was also performed with linear staplers. The gastrojejunostoma was closed by a hand-sewn technique, using the Endo Stitch ${ }^{\mathrm{TM}}$ suturing device. An adjustable gastric band (Ethicon) was used for the SITULAGBP procedure, performed with the pars flaccida method. A gastrogastric suture was performed using 2-0 Ethibond (Ethicon). After completing the procedure, the catheter was extracted from the 15 -mm port, connected to the injection port, and fixed in the paraumbilical area. In the SITU-LSG procedure, devascularization of the greater curvature was started $5 \mathrm{~cm}$ away from the pylorus and continued till the angle of Hiss, using the AutoSonix ${ }^{\mathrm{TM}}$ Ultra Shears ${ }^{\mathrm{TM}}$ Long Instrument (Covidien). The Fr36 calibration tube was retained as a stent for vertical gastrectomy using Endo GIA. After the completion of the main operative procedure, the LST was removed and hemostasis was achieved by cauterization. All trocars were removed and the surgical specimens were extracted into a plastic bag via the 12- or $15-\mathrm{mm}$ umbilical defect through which the trocars had been inserted. All the fascial defects were closed individually with 2-0 Vicryl sutures. Subsequently, an omega umbilicoplasty was performed such that the resultant wound was circular and hidden in the umbilicus (Fig. 3a-c). The surgical wound was dressed. The patients were transferred to the postoperative recovery room and then to the ward, provided their immediate postoperative course was uneventful. The patients were permitted to drink water and were discharged early if they did not develop any complications.

\section{Results}

A total of 40 consecutive patients were included in this 8 -month analysis (range, 1 to 8 months). The average body mass index (BMI) of these 40 bariatric patients was $40.1 \mathrm{~kg} / \mathrm{m}^{2}$ (range, $35.0-52.3 \mathrm{~kg} / \mathrm{m}^{2}$ ). The female-to-male ratio among the study patients was $32: 8$, and the mean patient age was 29 years. The most common comorbidity among the patients was hyperlipidemia (42.5\%), followed by nonalcoholic steatohepatitis (NASH; 27.5\%), hyperuricemia $(20.0 \%)$, hypertension $(17.5 \%)$, and type II diabetes mellitus $(15.0 \%)$. Preoperative ultrasonography revealed the presence of gallstones in five patients (12.5\%). One patient had bilateral osteoarthritis of the knees $(2.5 \%$; Table 2).
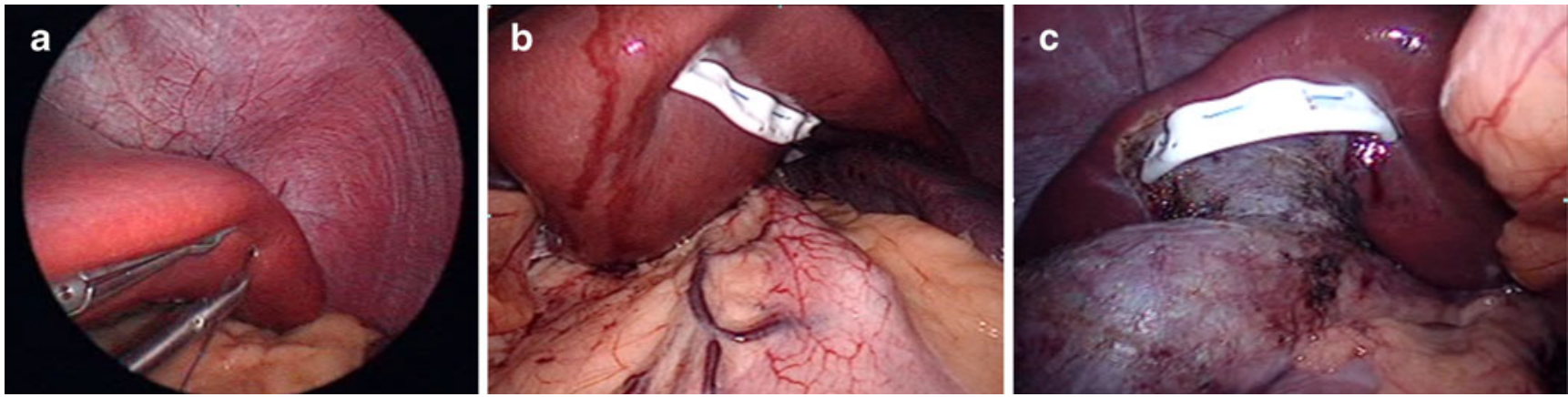

Fig. 2 Design for the application of the LST used for liver retraction. We measured the length of the left liver lobe intraoperatively and then cut a Jackson-Pratt drain tube to the same length near the site of the drainage hole. We penetrated it with a 2-0 prolene suture (monofilament polypropylene suture W8400; Ethicon). The needles were retained at both sides for further liver puncture. The liver-retraction procedure: a One of the needles attached to the LST was inserted into the left edge of the liver and then brought out through the abdominal wall in the left upper quadrant. b The other needle attached to the tape was inserted into the left liver lobe near the falciform ligament and then brought out through the abdominal wall in the midline. The liver was retracted to an appropriate position and the sutures fixed with Kelly clamps. c The right liver lobe suspended for retraction 
Fig. 3 Umbilicoplasty procedure: a repair the fascial defect, b umbilicoplasty, c circular wound repair. d Cosmetic outcome of the umbilical wound 3 months after the surgery
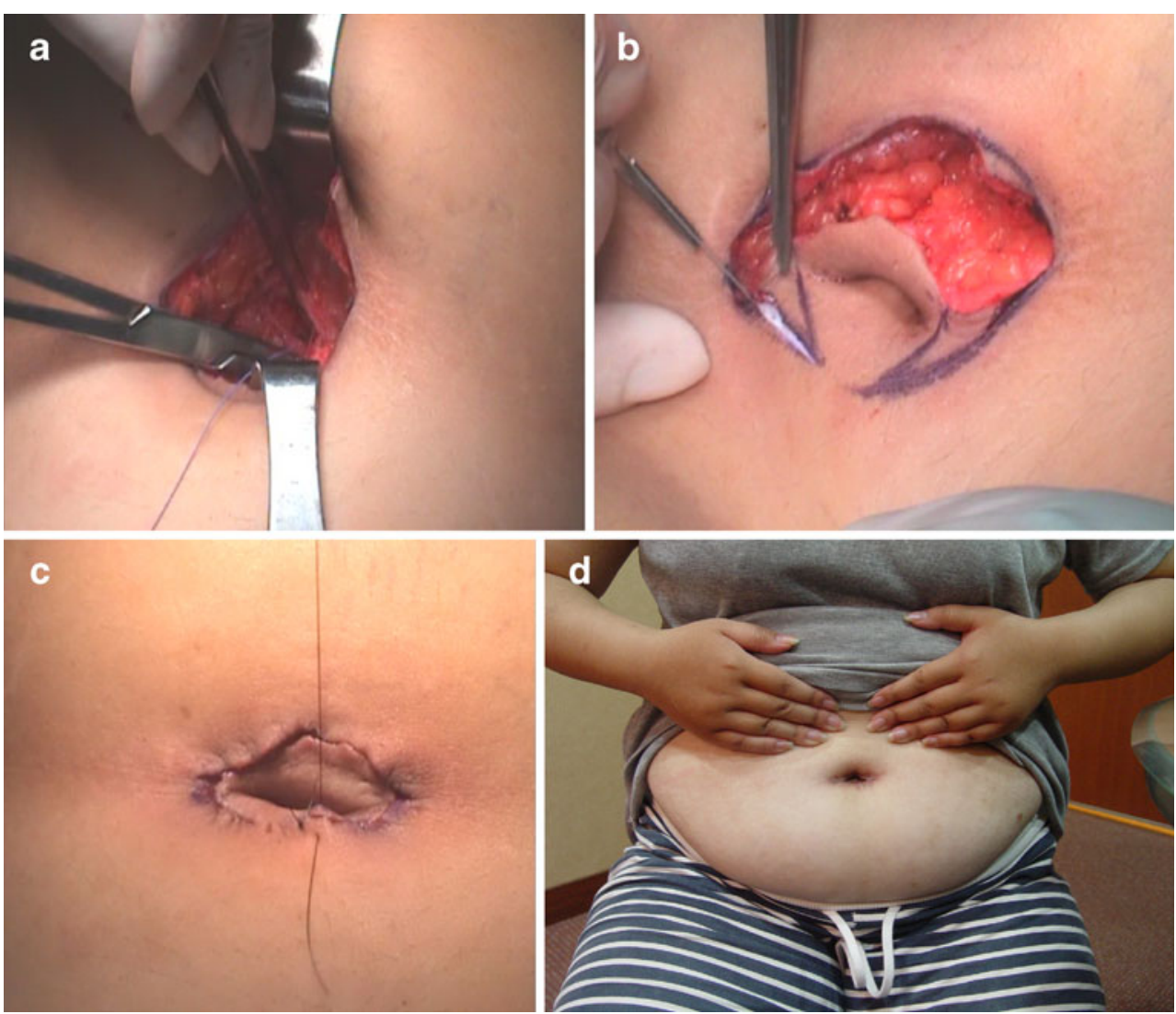

The mean operation time in these bariatric surgeries was $93.4 \mathrm{~min}$ (range, 37-144 min). The mean operation time for the most complicated surgery in our study, SITU-LRYGB, was $101.2 \mathrm{~min}$ (range, $81-144 \mathrm{~min}$ ). A total of 45 LSTs were used in the 40 patients: one each in 35 patients $(87.5 \%)$ and two each in the remaining five patients. LSTs were required in all the bariatric procedures, and two LSTs each were required to retract the hypertrophic left liver lobes in two patients and to retract the right liver lobe in three patients who underwent cholecystectomy. The mean time required for the placement of one LST was $5 \min 15 \mathrm{~s}$ (range, $1 \mathrm{~min} 41 \mathrm{~s}$ to $12 \mathrm{~min}$ ).
In the 40 patients, all the procedures were completed successfully without the need to convert to traditional laparoscopic surgery or use additional trocars. Thirty-four patients $(83.3 \%)$ were discharged within $24 \mathrm{~h}$ after the operation. The longest hospitalization period was 3 days, which was because the patient had postoperative fever.

We found that postoperative pain was indeed mild, and on an average, 2.5 (range, 0-6) morphine injections were required; moreover, six patients did not require morphine at all during the recovery stage. The mean wound satisfaction score at the third month after the procedures was 4.60 (range, 1-5).

Table 2 Operative results of SITU laparoscopic bariatric surgery

\begin{tabular}{llllll}
\hline Quartile & LRYGB $(n=32)$ & LAGBP $(n=2)$ & LSG $(n=6)$ \\
\hline Number of LST used & 35 & 3 & 7 & \\
Placement time for LST (mean) & $5 \min 29 \mathrm{~s} \pm 2 \min 35 \mathrm{~s}$ & $5 \min 9 \mathrm{~s} \pm 1 \min 10 \mathrm{~s}$ & $4 \mathrm{~min} 3 \mathrm{~s} \pm 1 \mathrm{~min} 4 \mathrm{~s}$ \\
Duration of surgery in minutes, mean (range) & $101.19(81-144)$ & $53.50(44-63)$ & $65.33(37-96)$ \\
Length of postoperative stay in days, mean (range) & $1.15(1-3)$ & $2(1-3)$ & 0 & $1(1)$ \\
Complications: wound seroma & 1 & $5(5)$ & $4.67(4-5)$ \\
Wound satisfaction score , mean (range) & $4.56(4-5)$ & & & 4 \\
\hline
\end{tabular}

Five patients underwent concomitant cholecystectomy and bariatric surgery

$L R Y G B$ laparoscopic Roux-en-Y gastric bypass, $L A G B P$ laparoscopic adjustable gastric band placement, $L S G$ laparoscopic sleeve gastrectomy

${ }^{\text {a }}$ Wound satisfaction score: very unsatisfied $=1$, unsatisfied $=2$, acceptable $=3$, satisfied $=4$, very satisfied $=5$ 
No major surgical complications occurred during follow-up, except the formation of wound seroma because of extensive subcutaneous dissection, which occurred in two patients $(5 \%)$. Most patients were very satisfied with the cosmetic outcomes (Fig. 3d).

\section{Discussion}

SILS was recently developed and is increasingly being used to perform various types of bariatric surgeries. Saber et al. reported the first SILS series in bariatric surgery, specifically transumbilical sleeve gastrectomies [7]. Because the surgery was performed via the transumbilical approach, the surgical scar was almost completely hidden inside the umbilicus. The main impediment to performing advanced laparoscopic surgery via the transumbilical approach is the crowding of instruments and the limited number of trocars that can be inserted in the narrow surgical field. The small degree of instrument triangulation complicates SITU laparoscopic surgery. We made a 6-cm omega-shaped incision around the upper half of the umbilicus to create a triangular opening and to increase the space available between the trocars. We found that, by using the above technique, the distance between the trocars was $4 \mathrm{~cm}$, which facilitated the maneuvering of the instruments. Furthermore, owing to our novel umbilicoplasty procedure, the omega-shaped incision was rendered circular and buried within the umbilicus. Our first patient was a woman with a BMI of $35.9 \mathrm{~kg} / \mathrm{m}^{2}$ who underwent SITU-LRYGB [15]. Initially, we simply made the 4-cm omega-shaped incision and closed it after the laparoscopic surgery. The operation time was $2 \mathrm{~h} 55 \mathrm{~min}$. After the introduction of omega umbilicoplasty, the operation time was dramatically decreased. Wound infection and suture dehiscence did not occur in this series. In two cases, we observed seroma formation because of the extensive dissection of subcutaneous fat. In order to avoid this complication, we suggest that the subcutaneous layer be approximated after fascia repair to minimize the dead space. The key aspect of the umbilicoplasty method is preserving the vascular pedicle in the 6 o'clock position and preventing ischemic changes. Therefore, we highly recommend minimal subcutaneous dissection around the umbilicus pedicle. Furthermore, we used a two-layer suture in the subcuticular layer and the skin to reduce the tension on skin healing, thus further preventing ischemia or necrosis.

With increasing improvement in and awareness of SILS, many surgeons are attempting to use this technique to perform more advanced surgeries. However, tissue retraction has to be performed by an assistant surgeon, and this is an obstacle in the development of this technique. Liver traction is critical for the feasibility and success of SILS. Sakaguchi et al. invented a device for the retraction of the liver during laparoscopic gastrectomy [16]. However, we found that their technique, which involves the dissection of the left triangular ligament of the left liver lobe, is difficult to use in morbidly obese patients because most of these patients have a hypertrophic left liver lobe. Our liver-retraction technique, which involves the use of LST, can be used to lift even massive livers in morbidly obese patients by using puncturing methods. Furthermore, if the liver is too heavy, the number of LSTs can be increased. Moreover, the time required for the placement of the LST was quite short: only $5 \min 15 \mathrm{~s}$ (range, $1 \mathrm{~min} 41 \mathrm{~s}$ to $12 \mathrm{~min}$ ). LST will also simplify laparoscopic bariatric procedures because it renders the retraction of the liver by an assistant. Furthermore, the LST could be easily removed after the completion of the laparoscopic procedure, and no hemorrhagic complications occurred after the cauterization of the liver puncture sites.

In our series, three super-obese patients were included and successfully underwent SITU-LRYGB. However, we found it very difficult to perform SITU in these super-obese patients. The BMI of our patients, including the three superobese patients, ranged from 35 to $52.3 \mathrm{~kg} / \mathrm{m}^{2}$. All patients had a very short postoperative hospital stay (mean, 1.15 days), and none of them developed any major postoperative complications. In all, we performed bariatric surgery using our novel technique in 40 patients, and all procedures were successfully completed without conversion to open surgery. The instruments, including the endoscope, that we used for the bariatric procedures were very long, up to $43 \mathrm{~cm}$. We believe that super obesity should not be a contraindication for SITU procedures; however, in these patients, preoperative abdominal assessment should be performed and the length of the laparoscopic instruments should be sufficient.

In our case series, no special articulating graspers, dissectors, or flexible endoscopes were needed. However, with the rapid evolution of MIS [17, 18], these instruments may be used during SITU laparoscopic surgery in the near future. Besides, we used the same technique to successfully perform splenectomy during the same study period. Therefore, we strongly believe that our novel technique is applicable to several surgeries besides bariatric ones.

\section{Conclusions}

We successfully performed SITU laparoscopic bariatric surgery (adjustable gastric band placement, sleeve gastrectomy, and RYGB) by using LSTs and omega umbilicoplasty with conventional laparoscopic instruments. We conclude that our laparoscopic technique is safe, feasible, and reproducible. 
Conflict of Interest Disclosure The authors declare that they have no conflict of interest.

Open Access This article is distributed under the terms of the Creative Commons Attribution Noncommercial License which permits any noncommercial use, distribution, and reproduction in any medium, provided the original author(s) and source are credited.

\section{References}

1. Chamberlain RS, Sakpal SV. A comprehensive review of singleincision laparoscopic surgery (SILS) and natural orifice transluminal endoscopic surgery (NOTES) techniques for cholecystectomy. J Gastrointest Surg. 2009;13:1733-40.

2. McGee MF, Rosen MJ, Marks J, et al. A primer on natural orifice transluminal endoscopic surgery: building a new paradigm. Surg Innov. 2006;13:86-93.

3. Flora ED, Wilson TG, Martin IJ, et al. A review of natural orifice translumenal endoscopic surgery (NOTES) for intra-abdominal surgery: experimental models, techniques, and applicability to the clinical setting. Ann Surg. 2008;247:583-602.

4. Tacchino R, Greco F, Matera D. Single-incision laparoscopic cholecystectomy: surgery without a visible scar. Surg Endosc. 2009;23:896-9.

5. Cuesta MA, Berends F, Veenhof AA. The "invisible cholecystectomy": a transumbilical laparoscopic operation without a scar. Surg Endosc. 2008;22:1211-3.

6. Reavis KM, Hinojosa MW, Smith BR, et al. Single-laparoscopic incision transabdominal surgery sleeve gastrectomy. Obes Surg. $2008 ; 18: 1492-4$.
7. Saber AA, Elgamal MH, Itawi EA, et al. Single incision laparoscopic sleeve gastrectomy (SILS): a novel technique. Obes Surg. 2008;18:1338-42.

8. Nguyen NT, Hinojosa MW, Smith BR, et al. Single laparoscopic incision transabdominal (SLIT) surgery-adjustable gastric banding: a novel minimally invasive surgical approach. Obes Surg. 2008;18 (12):1628-31.

9. Martínez AP, Bermejo MA, Cortś JC, et al. Appendectomy with a single trocar through the umbilicus: results of our series and a cost approximation. Cir Pediatr. 2007;20:10-4.

10. Barbaros U, Dinccaq A. Single incision laparoscopic splenectomy: the first two cases. J Gastrointest Surg. 2009;13:1520-3.

11. Bucher P, Pugin F, Morel P. Single port access laparoscopic right hemicolectomy. Int J Colorectal Dis. 2008;23:1013-6.

12. Irwin BH, Rao PP, Stein RJ, et al. Laparoendoscopic single site surgery in urology. Urol Clin North Am. 2009;36:223-35.

13. Canes D, Desai MM, Aron M, et al. Transumbilical single-port surgery: evolution and current status. Eur Urol. 2008;54:1020-9.

14. NIH Conference. Gastrointestinal surgery for severe obesity. Consensus Development Conference Panel. Ann Intern Med. 1991;115:956-61.

15. Huang CK, Houng JY, Chiang CJ, et al. Single-incision transumbilical laparoscopic Roux-en-Y gastric bypass: a first case report. Obes Surg. 2009;19:1711-5.

16. Sakaguchi $\mathrm{Y}$, Ikeda $\mathrm{O}$, Toh $\mathrm{Y}$, et al. New technique for the retraction of the liver in laparoscopic gastrectomy. Surg Endosc. 2008;22:2532-4.

17. Mintz Y, Horgan S, Savu MK, et al. Hybrid natural orifice translumenal surgery (NOTES) sleeve gastrectomy: a feasibility study using an animal model. Surg Endosc. 2008;22:1798-802.

18. Park S, Bergs RA, Eberhart R, et al. Trocar-less instrumentation for laparoscopy: magnetic positioning of intra-abdominal camera and retractor. Ann Surg. 2007;245:379-84. 